\title{
Correction of Varus Alignment with Peripheral Osteophyte Removal during Total Knee Arthroplasty: An Assessment with Computer Navigation
}

\author{
Nobuhiro Nishihara, MD ${ }^{1}$ Hironari Masuda, $\mathrm{MD}^{1}$ \\ Hirotaka Kawano, MD ${ }^{1}$ Takumi Nakagawa, MD ${ }^{1}$
${ }^{1}$ Department of Orthopaedic Surgery, Teikyo University School of Medicine, Tokyo, Japan
${ }^{2}$ Department of Orthopaedic Surgery, Shimazaki Hospital, Ibaraki, Japan

\author{
Naoya Shimazaki, MD ${ }^{1,2}$ Seikai Toyooka, MD ${ }^{1}$
}

J Knee Surg 2023;36:292-297.

\begin{abstract}
Address for correspondence Takumi Nakagawa, MD, Department of Orthopaedic Surgery, Teikyo University School of Medicine, 2-11-1 Kaga, Itabashi-ku, Tokyo 173-8606, Japan (e-mail: takumin-tky@umin.ac.jp).
\end{abstract}

\begin{abstract}
Keywords

- varus deformity

- peripheral osteophyte resection

- total knee arthroplasty

- hip-knee-ankle angle

Techniques for symmetrical balancing in flexion and extension have been described; however, the ideal technique is unclear. This study aimed to clarify whether resection of peripheral osteophytes could restore neutral hip-knee-ankle (HKA) angle of varus deformity of arthritic knees. Data from 90 varus arthritic knees that had undergone total knee arthroplasty (TKA) using a nonimage-based navigation system were analyzed. The change in the coronal mechanical axis, while applying manual valgus stress at extension and 90 degrees of knee flexion, was recorded after the following sequential procedures: (1) anterior cruciate ligament (ACL) sectioning, (2) subperiosteal stripping of the deep medial collateral ligament $(\mathrm{MCL})$ from the underlying osteophytes on the medial tibia, and (3) complete removal of peripheral osteophytes from the proximal medial tibia and distal medial femoral condyle. Repeated measures of analysis of variance (ANOVA) were performed to compare the varus angle among each step, and a post hoc analysis by paired $t$-test was utilized to compare the parameters between baseline and each step. The varus alignment with valgus stress at extension and 90 degrees of flexion (mean: $6.0 \pm 3.6$ and $5.2 \pm 3.9$ degrees of varus, respectively) was significantly corrected to a near-neutral mechanical axis (mean: $0.9 \pm 2.4$ and $1.4 \pm 4.2$ degrees of varus, respectively) after peripheral osteophyte resection ( $p<0.01$, both). In many cases, varus deformity of arthritic knees could be corrected to near-neutral HKA angle by applying manual valgus stress after complete peripheral osteophyte resection. These procedures could facilitate soft tissue balancing in TKA, minimizing the risk of overrelease of the medial soft tissues.
\end{abstract}

The main surgical requirements for restoring optimal function in total knee arthroplasty (TKA) are an accurate bone cut to achieve alignments and soft tissue balancing throughout flexion. ${ }^{1-5}$ Balancing has been described as equal and rectangular gaps between the resected bone surfaces in both extension and flexion, implying that the lateral and medial

received

January 20, 2021

accepted

May 31, 2021

article published online

July 16, 2021

soft tissues maintain equal tensions. Incorrect soft tissue balancing can result in several complications, including instability, abnormal polyethylene wear, aseptic loosening, altered patellofemoral biomechanics, and pain. ${ }^{6,7}$ Although the standard of care is to balance the gaps symmetrically in both flexion and extension, and multiple techniques have

(c) 2021. Thieme. All rights reserved. Thieme Medical Publishers, Inc., 333 Seventh Avenue, 18th Floor, New York, NY 10001, USA
DOI https://doi.org/ 10.1055/s-0041-1731737. ISSN 1538-8506. 

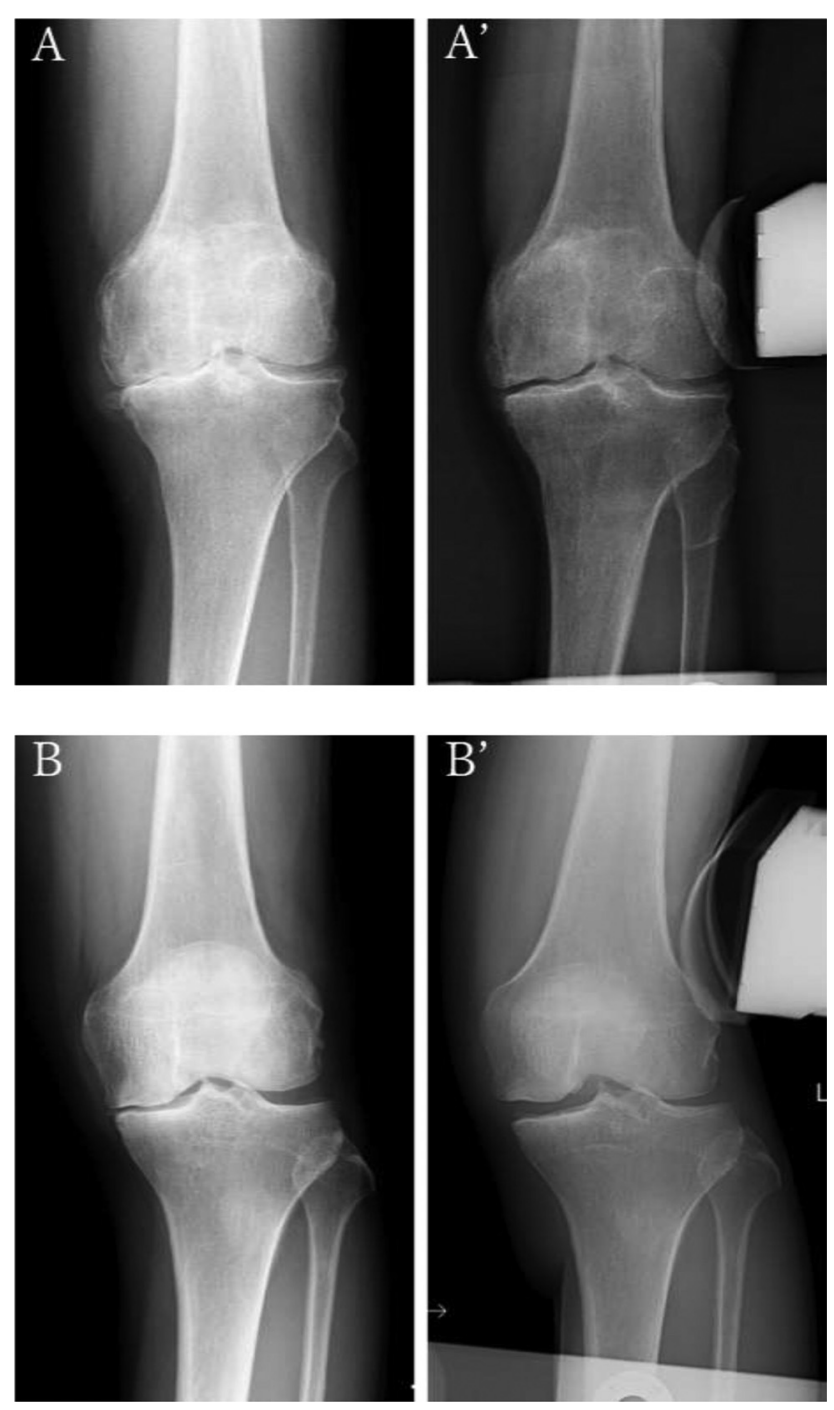

Fig. 1 Anteroposterior radiograph of the left knee in the neutral position and under valgus stress. The varus deformity is fixed in case 1 ( $A$ and $A^{\prime}$ ), while the varus deformity is corrected to near-normal alignment in case 2 ( $B$ and $\mathbf{B}^{\prime}$ ).

been described to achieve this surgical objective, there is no consensus on the ideal ligament balancing technique. ${ }^{8-12}$

Recently, it has been suggested that knee varus deformity can be classified as correctable or fixed. ${ }^{13}$ In fixed varus deformity, the coronal alignment of the knee cannot be corrected to neutral in preoperative stress radiographs (-Fig. 1). Correction of medial soft tissue tightness in severe varus osteoarthritis (OA) may require extensive medial soft tissue release to balance the knee at the time of TKA which is generally performed after femoral and tibial bone cuts. Occasionally, the release of the superficial medial collateral ligament (MCL) from the proximal medial aspect of the tibia with or without the release of the pes anserinus insertion is also required. ${ }^{14-17}$ Unfortunately, this can lead to overrelease of the superficial MCL, hematoma formation, knee joint line elevation, instability, and the need for constrained TKA implants. ${ }^{14,16-20}$ If the severe varus alignment was to be corrected with valgus manual stress to a near-neutral mechanical axis before bone cuts, it could facilitate the achievement of adequate soft tissue balancing without the need for any additional soft tissue work. Bellemans et al reported that knees with $<10$ degrees of varus deformity were easily correctable to neutral at extension after correction of the extrinsic factors in their navigation-assisted TKA study. ${ }^{21}$ In their study, the correction of preoperative varus deformity was investigated by interposing a specially developed intercalary shim between the medial compartment defects. To elucidate if removal of the extrinsic factors could correct fixed varus deformity both at extension and 90 degrees of knee flexion in the Asian population, uniform subperiosteal release from the medial aspect of tibia and the removal of peripheral osteophytes formed at medial joint space were sequentially performed before any bone cuts in navigation-assisted TKA for the varus OA knee. Instead of interposing a specially developed device to fill the cartilage and bone defect in the medial compartment, manual valgus stress was applied both at knee extension and 90 degrees of flexion, and the hip-kneeankle (HKA) angle of the knee was recorded using the computer navigation. This study aimed to determine the contribution of uniform release of soft tissue attachment to expose peripheral osteophytes and osteophyte removal to the magnitude of the passive mechanical axis of extension and flexion in preparation for a TKA, using an image-free computer navigation system. Our hypothesis was that severe preoperative varus alignment could be corrected to a near-neutral mechanical axis before bone cuts after uniform release of the deep MCL and complete removal of peripheral osteophytes during TKA in varus $\mathrm{OA}$.

\section{Materials and Methods}

Ninety consecutive patients undergoing TKA with computerassisted navigation for varus knee arthritis from August 2015 to November 2017 were included in this study. Inclusion criteria were varus OA knees with a Kellgren-Lawrence arthritis classification of 3 degrees or more. Exclusion criteria were patients with rheumatoid arthritis (RA) and patients with any history of surgery on the affected leg. All patients were informed regarding the procedure and provided consent for participation in the study and the publication of their data. The institutional review board approved this study. The patient population was composed of 72 (80\%) women and 18 (20\%) men, with a mean age of 76.7 (range: 63-96) years. The degree of arthritis in the operated knees was classified using the Kellgren-Lawrence arthritis classification based on preoperative standing anteroposterior radiographs. All patients underwent full-leg standing anteroposterior radiographs preoperatively to analyze the HKA angle ( - Table $\mathbf{1}$ ). They were under the care of three experienced surgeons ( $T$. N., H.M., and N.N.) who use computer navigation routinely in their standard TKA practice. The knee navigation system (Ortho Map Precision Knee Navigation Software ver4.0, Stryker, Mahwah, NJ) was used and is based on a principle of hip, knee, and ankle center acquisition via kinematic and anatomical landmark registration processes.

After knee exposure, using the medial parapatellar or midvastus approach, active patient trackers were fixed 
Table 1 Preoperative patient characteristics

\begin{tabular}{|l|l|}
\hline Characteristics & Values \\
\hline Number of cases & 85 \\
\hline Number of knees & 90 \\
\hline Age (range) & $76.7(63-96)$ years \\
\hline Sex (male/female) & $18 / 72$ \\
\hline Body mass index & $25.9 \pm 3.8 \mathrm{~kg} / \mathrm{m}^{2}$ \\
\hline FTA (degrees) & $186.5 \pm 4.1$ \\
\hline HKA angle (degrees) & $12.3 \pm 4.6$ \\
\hline ROM (degrees) & \\
\hline Extension & $-6.8 \pm 7.8$ \\
\hline Flexion & $117.4 \pm 17.9$ \\
\hline KL & \\
\hline Grade III & 6 \\
\hline Grade IV & 84 \\
\hline
\end{tabular}

Abbreviations: FTA, femoral-tibia angle; HKA, hip-knee-ankle angle; $\mathrm{KL}$, Kellgren-Lawrence; ROM, range of motion.

onto the femur and tibia with two bicortical anchoring pins on each bone. Surgical exposure of the joint was performed to allow the patient's anatomy to be registered according to standard procedure. After the registration of anatomical landmarks owing to the standard navigation workflows, coronal plane alignment represented by the femorotibial mechanical angle was recorded in both extension and 90 degrees of flexion, both in the neutral position (no applied stress) and at maximum valgus stress.

Initially, no ligamentous releases or osteophyte removals were performed, and the value was documented as the baseline HKA angle (step 0 ). Thereafter, the following procedures were performed sequentially: step 1 , sectioning of the anterior cruciate ligament (ACL) if it was present (if the ACL was torn out due to friction with osteophytes at the exit of the femoral notch, this step was skipped); step 2, elevation of a periosteal sleeve down to approximately $5 \mathrm{~mm}$ distal to the joint line including part of deep MCL fibers from the medial aspect of the tibia; and step 3, complete removal of peripheral osteophytes from the medial and posteromedial aspect of the proximal tibia and distal medial femoral condyle. All reachable osteophytes were carefully removed, and the tibia was subluxated anteriorly while applying maximum external rotation torque to the tibia to provide access to the osteophyte built on the periphery of the posteromedial corner of the medial tibial plateau (-Fig. 2). The femorotibial mechanical angle with maximum manual valgus stress was recorded at each step on the computer navigation system, and the angle in neutral position was recorded at step 0 and step 3. Moreover, the femorotibial mechanical angle in neutral position after implantation was recorded. All measurements were performed by surgeons with high-volume experience in TKA. Data for all patients were extracted from these navigation files and were used to
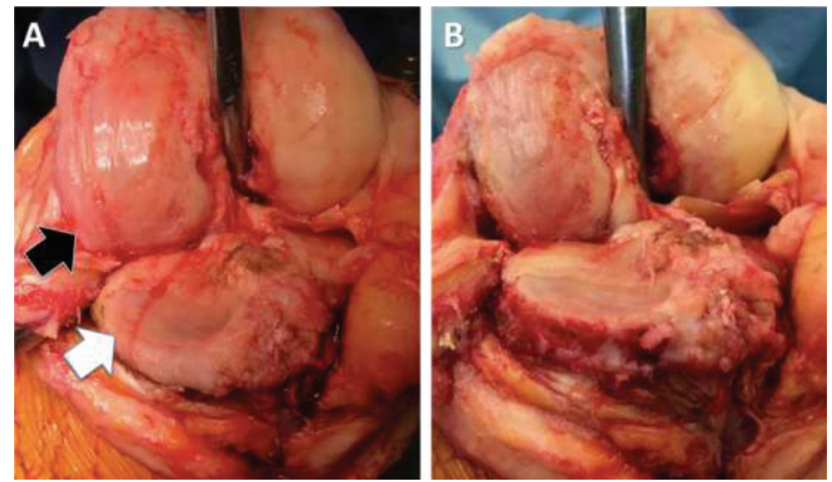

Fig. 2 All reachable osteophytes from the medial and posteromedial aspect of the (A) proximal tibia (white arrow) and distal medial femoral condyle (black arrow) are carefully removed (B).

quantify the effect of each step on varus alignment of the knee.

\section{Statistical Analysis}

All statistical analyses were performed using SPSS version 21 (IBM Corp, Armonk, NY). Repeated measures of analysis of variance (ANOVA) were performed to compare the varus angle among each step, and a post hoc analysis by paired $t$-test was utilized to compare the parameters between baseline and each step. Statistical significance was defined as $p<0.05$.

\section{Results}

The mean baseline HKA angle recorded in the neutral position was $10.1 \pm 4.2$ degrees of varus (range: $4-20.5$ degrees of varus) at extension and $7.2 \pm 3.5$ degrees of varus (range: 0.5-15.5 degrees of varus) at 90 degrees of flexion. At step 3 , the mean HKA angle recorded in the neutral position was $9.5 \pm 3.9$ degrees of varus (range: $2-19.5$ degrees of varus) at extension and $6.0 \pm 3.8$ degrees of varus (range: 0 16 degrees of varus) at 90 degrees of flexion. The mean baseline femorotibial mechanical axis recorded with manual valgus stress was $6.0 \pm 3.6$ degrees of varus (range: -1 to 20.5 degrees of varus) at extension and $5.2 \pm 3.9$ degrees of varus (range: -2 to 15.5 degrees of varus) at 90 degrees of flexion. At step 1, the alignment with valgus stress at extension and 90 degrees of of flexion was $5.4 \pm 3.2$ and $4.9 \pm 3.9$ degrees of varus, respectively. At step 2, the alignment with valgus stress at extension and $90^{\circ}$ of flexion was $4.7 \pm 3.2$ and $3.9 \pm 4.0$ degrees of varus, respectively. The mean passive varus alignment with valgus stress was corrected to $1.0 \pm 2.5$ degrees of varus (range: -4.5 to 10 degrees of varus) at extension and $1.4 \pm 4.2$ degrees of varus (range: -10.5 to 12.5 degrees of varus) at 90 degrees of flexion after complete resection of the peripheral osteophytes ( $p<0.01$, both vs. baseline; -Fig. 3 ). The passive mechanical axis with valgus stress in knee extension was corrected to $<3$ degrees of varus in $84.4 \%$ of cases in extension and $70 \%$ of cases in 90 degrees of knee flexion (-Fig. 4). The mean HKA angle in the neutral position right after implantation was $0.8 \pm 1.2$ degrees in 
A

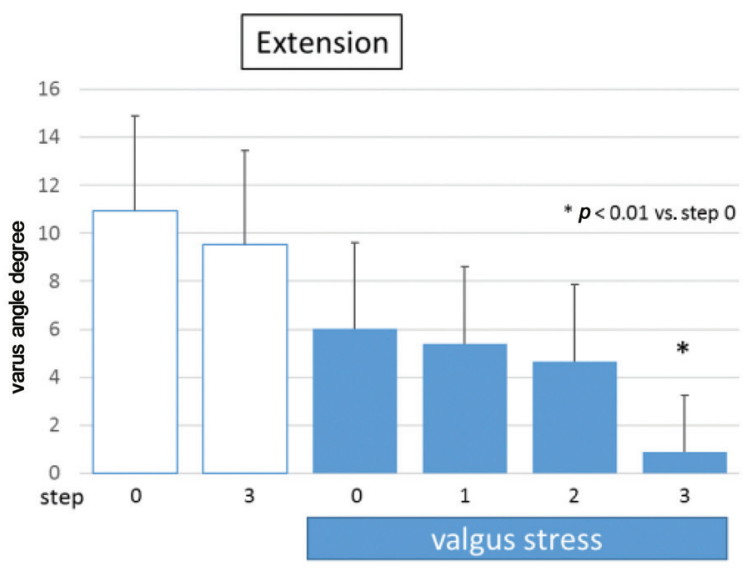

B

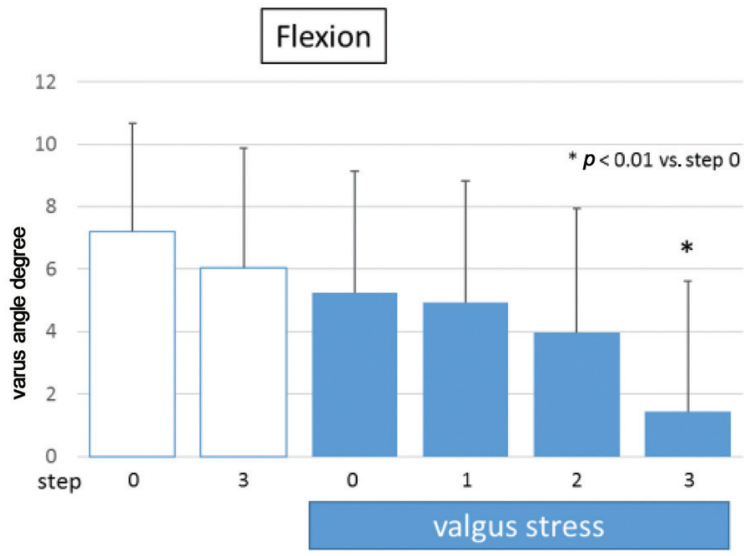

Fig. 3 The HKA angle with and without valgus stress after each procedure in extension (A) and flexion (B). The white and blue bars show the HKA angle in the neutral position and with valgus stress, respectively. The HKA angle with valgus stress is significantly restored both in extension and flexion after the complete resection of the peripheral osteophytes. HKA, hip-knee-ankle.

extension and $0.5 \pm 1.4$ degrees in flexion. $96.7 \%$ of cases were within 3 degrees of varus and valgus ( - Fig. 5).

\section{Discussion}

The most important finding of this study was that even if it was fixed varus deformity, it could be corrected to a nearneutral HKA angle while applying manual valgus stress, not after subperiosteal elevation of the medial tibial plateau but after complete resection of osteophytes at both the medial and posteromedial edges of the tibial plateau and distal medial femoral condyle before any bone resections during TKA for varus OA. In the current study, medial release and osteophyte removal were performed in the same sequential manner, and the HKA angle of the knee was evaluated objectively with the assistance of the image-free navigation system. ${ }^{22}$ Based on the results from the sequence of the procedures, resection of the peripheral osteophytes, which tents up the deep and superficial MCL or posteromedial capsule, seems critical to the correction of the fixed varus deformity. These results are in accordance with those in the study by Bellemans et al wherein knees with $<10$ degrees of

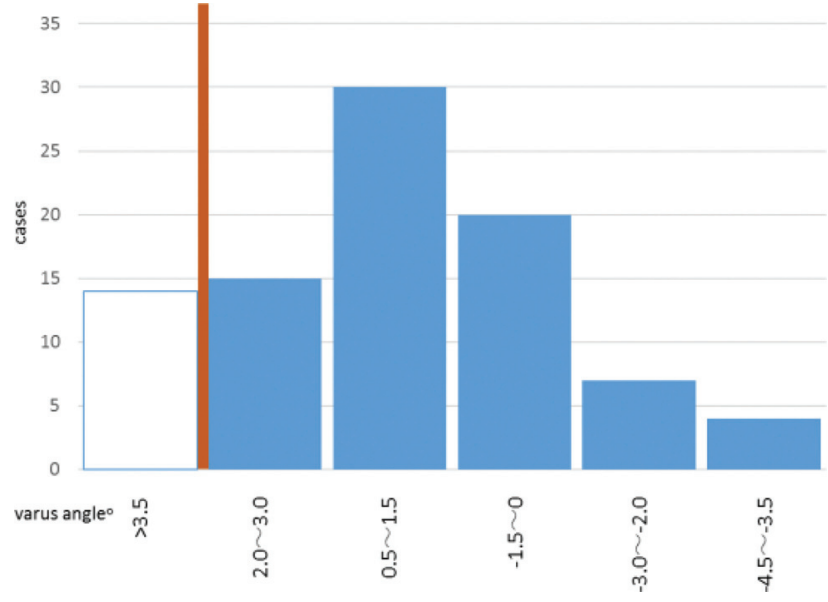

Fig. 4 A histogram for the HKA angle with valgus stress in extension after complete resection of the peripheral osteophytes (step 3 ). The white bar shows the cases wherein alignment was $>3$ degrees of varus.

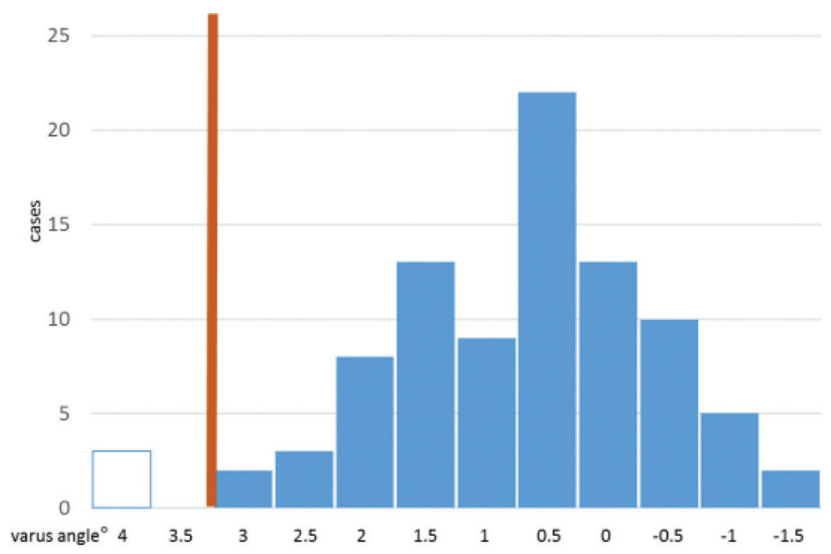

Fig. 5 A histogram for the HKA angle in the neutral position after implantation. The white bar shows the cases wherein alignment was $>3$ degrees of varus.

varus deformity were easily corrected to neutral after the correction of the extrinsic factors that could cause medial tightness. $^{21}$

Fixed varus deformity of the knee is associated with bone loss in the tibia and medial femoral condyle, leading to severe varus malalignment secondary to contracture of the medial soft tissue structures. Recently, Thienpont and Parvizi proposed a new classification for the varus knee that allows for a better definition of varus deformity. ${ }^{13}$ According to their classification, varus deformity can either be intra-articular or extra-articular, and intra-articular deformities can be further classified into correctable or fixed. In our current case series, the passive HKA angle recorded in manual valgus stress after the initial registration was corrected to $<3$ degrees of varus in 20 knees (22.2\%) and was classified as correctable varus deformity. Therefore, many TKA cases in this study presented with fixed varus deformity that needed to be corrected intraoperatively. From an unbalanced state, balancing can be achieved in different ways, including soft tissue release, making small modifications to bone cuts, and changing component sizes. Soft tissue release is the most 
common procedure and can be easily performed, although it is less quantitative. Moreover, the procedure requires some experience to be safely performed and is accompanied by the risk of instability. A biomechanical study on cadaveric specimens showed great variability in elongation and failure of the MCL after pie-crusting technique. ${ }^{23}$ However, peripheral osteophyte resection is a relatively simple surgical procedure and can be routinely performed even by less experienced surgeons. Here, the passive mechanical axis with valgus stress in knee extension was corrected to $<3$ degrees of varus in $84.4 \%$ of cases in extension and $70 \%$ of cases in 90 degrees of knee flexion after complete removal of peripheral osteophytes. Additionally, the final mechanical axis of the knee after implantation was achieved within 3 degrees of varus and valgus in $96.7 \%$ of the cases. Hence, we recommend peripheral osteophyte removal initially from both the medial femoral condyle and medial aspect of the tibia before any bone cut as one effective method. This method allows us to understand the soft tissue balance of each patient at the beginning of the procedure, and as a result, it achieves good lower limb alignment in TKA. Once the tightness of medial soft tissue envelop is normalized after these procedures in TKA for varus OA, an appropriate soft tissue balance could relatively easily be achieved without the need for any additional soft tissue work in many cases. It also leads to the preservation of attachment and midsubstance fibers of superficial MCL and the attachments of pes anserinus and semimembranosus, thereby contributing to a well-functioning pain-free knee joint, without the risk of medial soft tissue overrelease.

The sequential procedures performed in this study were not specially designed protocols, but rather the regular workflows used in both cruciate-retaining and posteriorstabilized TKA implantation. After the arthrotomy was performed, the ACL was initially sectioned, while the posterior cruciate ligament was untouched. To subluxate the tibia anteriorly, minimum subperiosteal elevation of deep MCL fibers and the joint capsule from the medial and posteromedial aspects of the tibia was inevitable. The deep MCL was minimally liberated from the underlying osteophytes on the medial tibia. All reachable osteophytes on the medial femur and tibia were carefully removed. Although osteophyte removal from the femur is not challenging, care was taken not to damage the insertion of the superficial MCL at the medial epicondyle. The removal of the osteophytes from the posteromedial aspects of the tibia was performed with the tibia subluxated at maximum external rotation, and care was taken not to damage the semimembranosus insertion.

\section{Limitations}

Our study has some limitations inherent in the measurement techniques used. Manual stress testing was performed to assess mediolateral laxity, and despite being performed by experienced surgeons, some degree of variability in the forces exerted during testing cannot be excluded. Moreover, all measurements were to be performed with the knee in full extension ( 0 degrees of flexion); however, this was not possible in all cases. Cases with flexion contractures were analyzed with the knees in the fully extended position and not at 0 degrees of flexion. However, the use of modern navigation may enable the intraoperative quantification of mediolateral laxity of the knee more accurately and reproducibly compared to any other available alternative. Although the proportion of correctable varus knees after resection of the osteophytes was shown in this study, the relationship between the correction of fixed varus deformity and preoperative varus deformity remains unclear. Further study will be needed to clarify whether fixed varus deformity is corrected or uncorrected depending on the preoperative varus deformity.

\section{Conclusion}

Our study demonstrated that varus contracture of arthritic knees could be corrected to near-neutral mechanical axis after complete resection of the peripheral osteophytes in many cases. These procedures may facilitate soft tissue balancing in TKA, while decreasing the need for additional soft tissue work and minimizing the risk of overrelease of the medial soft tissues as a result.

Conflict of Interest

None declared.

\section{References}

1 Insall J, Tria AJ, Scott WN. The total condylar knee prosthesis: the first 5 years. Clin Orthop Relat Res 1979;(145):68-77

2 Insall JN, Binazzi R, Soudry M, Mestriner LA. Total knee arthroplasty. Clin Orthop Relat Res 1985;(192):13-22

3 Dorr LD, Boiardo RA. Technical considerations in total knee arthroplasty. Clin Orthop Relat Res 1986;(205):5-11

4 Whiteside LA, Saeki K, Mihalko WM. Functional medical ligament balancing in total knee arthroplasty. Clin Orthop Relat Res 2000; (380):45-57

5 Unitt L, Sambatakakis A, Johnstone D, Briggs TWBalancer Study Group. Short-term outcome in total knee replacement after softtissue release and balancing. J Bone Joint Surg Br 2008;90(02): 159-165

6 Fehring TK, Odum S, Griffin WL, Mason JB, Nadaud M. Early failures in total knee arthroplasty. Clin Orthop Relat Res 2001; 392(392):315-318

7 Sharkey PF, Hozack WJ, Rothman RH, Shastri S, Jacoby SM. Insall Award paper. Why are total knee arthroplasties failing today? Clin Orthop Relat Res 2002;(404):7-13

8 Chen W, Nagamine R, Kondo K, Todo M. Effect of medial softtissue releases during posterior-stabilised total knee arthroplasty. J Orthop Surg (Hong Kong) 2011;19(02):230-233

9 Chon JG, Sun DH, Jung JY, Kim TI, Jang SW. Rotational alignment of femoral component for minimal medial collateral ligament release in total knee arthroplasty. Knee Surg Relat Res 2011;23(03): 153-158

10 Moon YW, Kim JG, Han JH, Do KH, Seo JG, Lim HC. Factors correlated with the reducibility of varus deformity in knee osteoarthritis: an analysis using navigation guided TKA. Clin Orthop Surg 2013;5(01):36-43

11 Mullaji AB, Padmanabhan V, Jindal G. Total knee arthroplasty for profound varus deformity: technique and radiological results in 173 knees with varus of more than 20 degrees. J Arthroplasty 2005;20(05):550-561 
12 Hunt NC, Ghosh KM, Athwal KK, Longstaff LM, Amis AA, Deehan DJ. Lack of evidence to support present medial release methods in total knee arthroplasty. Knee Surg Sports Traumatol Arthrosc 2014;22(12):3100-3112

13 Thienpont E, Parvizi J. A new classification for the varus knee. J Arthroplasty 2016;31(10):2156-2160

14 Laskin RS. The Insall Award. Total knee replacement with posterior cruciate ligament retention in patients with a fixed varus deformity. Clin Orthop Relat Res 1996;(331):29-34

15 Teeny SM, Krackow KA, Hungerford DS, Jones M. Primary total knee arthroplasty in patients with severe varus deformity. A comparative study. Clin Orthop Relat Res 1991;(273):19-31

16 Engh GA. The difficult knee: severe varus and valgus. Clin Orthop Relat Res 2003;(416):58-63

17 Engh GA, Ammeen D. Results of total knee arthroplasty with medial epicondylar osteotomy to correct varus deformity. Clin Orthop Relat Res 1999;(367):141-148

18 Parratte S, Pagnano MW. Instability after total knee arthroplasty. J Bone Joint Surg Am 2008;90(01):184-194
19 Abdel MP, Haas SB. The unstable knee: wobble and buckle. Bone Joint J 2014;96-B(11(supple A):112-114

20 Mullaji AB, Shetty GM. Correcting deformity in total knee arthroplasty: Techniques to avoid the release of collateral ligaments in severely deformed knees. Bone Joint J 2016;98-B(01(suppl A):101-104

21 Bellemans J, Vandenneucker H, Vanlauwe J, Victor J. The influence of coronal plane deformity on mediolateral ligament status: an observational study in varus knees. Knee Surg Sports Traumatol Arthrosc 2010;18(02):152-156

22 Hakki S, Coleman S, Saleh K, Bilotta VJ, Hakki A. Navigational predictors in determining the necessity for collateral ligament release in total knee replacement. J Bone Joint Surg Br 2009;91 (09):1178-1182

23 Amundsen SH, Meyers KN, Wright TM, Westrich GH. Variability in elongation and failure of the medial collateral ligament after piecrusting with 16- and 18-gauge needles. J Arthroplasty 2018;33 (08):2636-2639 